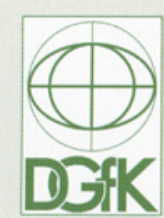

KN Kartographische Nachrichten Journal of Cartography and Geographic Information 67. Jahrgang, Dezember 2017, Heft 6 Herausgeber: Deutsche Gesellschaft für Kartographie e.V. (DGfK) - Gesellschaft für Kartographie und Geomatik, vertreten durch den Präsidenten durch den Präsidenten
Prof. Dr. Manfred Weisensee

Organ der Deutschen Gesellschaft für Kartographie e.V. (DGfK). der Schweizerischen Gesellschaft für Kartografie (SGK) und der Österreichischen Kartographischen Kommission (ÖKK) in der

Österreichischen Gesellschaft für Geographie (ÖGG).

Schriftleitung

Hauptschriftleiter

Prof. Dr. rer. nat. habil. Mark Vetter (zuständig für Aufsätze, Berichte). Hochschule Karlsruhe - Technik und Wirtschaft. Moltkestr. 30, 76133 Karlsruhe: redaktion @kartographischenachrichtende, Fon:0049(0) 7219252599

Weitere Schriftleiter

Prof. Dr. phil. Frank Dickmann (Zweiter Schriftleiter, zuständig für Nachrichten aus Hochschulen und Institutionen, Rezensionen).

Ruhr-Universität Bochum, Geographisches Institut,

Postfach, 44780 Bochum; frank.dickmann@ @rub.de

Fon: 0049(0) 234 32-23379; Fax: 0049(0) 234 32-14964

Dipl.-Ing. Andreas Gollenstede (zuständig für Geoinformation

aktuell) Haareneschstr. 91, 26121 Oldenburg; kn@gollenstedecom

Fon: 0049(0) $4417779-545$; Fax: 0049(0) 4417779-750

Dr.-Ing. Thomas Chudy (zuständig für DGfK-, SKG- und ŐGG-

Nachrichten). Martin-Luther-Universităt Halle-Wittenberg, Institut

für Agrar- und Ernährungswissenschaften, Karl-Freiherr-von-

Eritsch-Straße 4, 06120 Halle/Saale, Fon: 0049(0) 3455522448 .

Fax: 0049(0) 3455527109

Prof. Dr. Reinhard Zölitz (zuständig für Nachrichten aus Hoch

schulen und Institutionen), Institut für Geographie und Geologie,

Kartographie und GIS, Friedrich-Ludwig-Jahn-Str. 16,

D-17489 Greifswald, Fon 03834864523, Fax: 03834864501,

E-Mail:zoelitz@uni-greifswald.de

Mitarbeit für die Schweiz: Prof. Dr. Lorenz Hurni. Institut für

Kartografie, ETH Hönggerberg, 8093 Zürich; hurni@karto.baug.ethz.

ch. Fon: 0041 16333033; Fax:0041 1631153

Mitarbeit für Österreich: Univ.-Prof. Dr. Wolfgang Kainz

Institut für Geographie und Regionalforschung, Universität Wien,

Universitätsstraße 7/5, 1010 Wien; wolfgang.kainz@univie.ac.at

Fon: 0043 14277-8640; Fax: 0043 14277-9531

Ehrenschriftleiter

Prof. Dr. Jürgen Dodt $t$, Witten

Dr. rer. nat. h. c. Rolf Harbeck, Bonn

Editorial Board

Prof. Dr. H. Asche, Potsdam; Prof. Dr. Manfred Buchroithner

Dresden; Prof. Dr. Dirk Burghardt, Dresden; Prof. Dr. D. Dransch,

Potsdam; Prof. Dr. M. Ehlers, Osnabrück, Berlin; Prof. Dr. S.

Fabrikant, Zürich, Schweiz; Ass-Prof. Dr. S. Fuhrmann. Fairfax

USA:Prof. Dr: G. Gertner. Wien. Osterreich: Prof. Dr. D. Grünreich,

USA, Prof. Dr. G. Gartner, Wien, Osterneich, Prof. Dr. D. Grunreich,

Ronnenberg; Dr. Rolf Harbeck, Bonn, Prof. Dr. A. Hüttermann,

Prof Dr. K, Yrof. Dr. L. Humi, Zunich, Prof. Dr. W. Kainz, Wien:

Prof. Dr. P. Kammerer, Munchen; Prof. Dr. W. G. Koch, Dresden;

Prof. Dr. Menno-Jan Kraak, Twente, Netherlands; Prof. Dr.

Jukka Krisp, Augsburg; Prof. Dr.-Ing. L Meng, München;

Prof. Dr. J. Schiewe, Hamburg; PD Dr.-Ing. J. Schoppmeyer, Bonn:

Prof. Dr. J. Schweikart, Berlin; Prof. Dr.-Ing. M. Sester, Hannover:

Prof. Dr. J. Siemer, Regina, Kanada; Prof. Dr. R. Zölitz, Greifswald.

Manuskripte richten Sie bitte an den zustāndigen Schriftleiter.

Hinweise zur Gestaltung des Manuskripts finden Sie unter

www.kartographische-nachrichten.de > Autorenhinweise.

Reviewverfahren

Wissenschaftliche Beiträge werden im Rahmen eines Peer-ReviewVerfahrens begutachtet. Trotz sorgfältiger Redigierung übernehmen Herausgeber. Redaktion und Verlag keine Haftung für die inhalttiche Richtigkeit der Veröffentlichungen.

Die KN Kartographische Nachrichten ist in der internationalen

Zitationsdatenbank Scopus gelistet.

Die DGfK ist Mitglied der

Internationalen Kartographischen

Vereinigung IKV/ICA.

Verlag: Kirschbaum Verlag GmbH

Siegfriedstraße 28, D-53179 Bonn

Postfach 210209, D-53157 Bonn

Fon: (0228) 95453-0, Fax: (0228) 95453-27

Internet: http://www.kirschbaum.de, E-Mail: info@kirschbaum.de

Anzeigenleiter: Michael Dietl Nerlagsleiter Fachzeitschriften prin

Et digital), Fon: (0228) 95453-47, E-Mail: m.dietl@kirschbaum.de;

Anzeigenpreisliste Nr. 53 vom 1.10.2017

Bezugspreise und ISSN: Inland/Ausland Jahresabonnement

inkl. E-Paper und elektronischem Archiv 74,- Euro zzgl.

Versand 9,80,- Euro (jeweils einschl. MwSt.).

Einzelheft 18,- Euro zzgl. Versand

Die Zeitschrift erscheint alle zwei Monate. ISSN 0022-9164.

Kündigungsfrist 6 Wochen zum Auslaufen des Abonnement-

Zeitraumes.

Satz: EMS Eckert Medienservice, 53881 Euskirchen

Druck: johnen-druck GmbH \& Co. KG, Industriegebiet Bornwiese,

54470 Bernkastel-Kues

\section{Kartenarbeit in Archiven}

\section{Liebe Leserin, lieber Leser,}

für die wissenschaftliche Arbeit mit historischen Karten ist man darauf angewiesen, die verwahrenden Institutionen (zumeist Bibliotheken oder Archive) aufzusuchen. Glücklicherweise finden sich in Europa zahlreiche, hervorragende Bibliotheken, aus deren Quellen ganz unterschiedliche Zeiträume und Themen betrachtet werden können. Als herausragendes Beispiel ist hier sicherlich die Kartenabteilung der Staatsbibliothek zu Berlin $\mathrm{zu}$ nennen; die überregionale Bedeutung der wertvollen Bestände dieser Sammlung hebt die Generaldirektorin, Barbara Schneider-Kempf, auf den Seiten 342-345 dieser

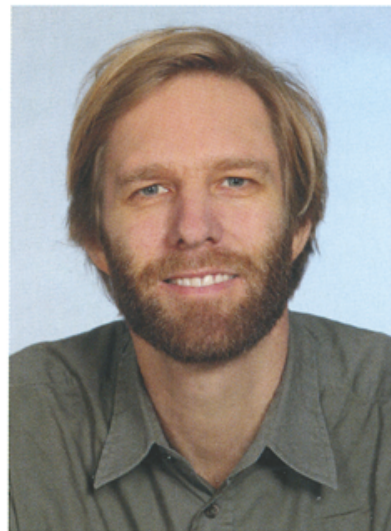

Mark Vetter Ausgabe hervor. Die Zugänglichkeit zu den historischen Plänen und Karten ist in der Regel kein Problem, und gute Bibliotheken verfügen ferner über ausgewiesene Fachexperten, bei denen man einen zielführenden, wissenschaftlichen Rat bekommen kann.

Freilich sollen die Erkenntnisse, die in den Bibliotheken und Archiven von den dort tätigen Wissenschaftlerinnen und Wissenschaftlern gewonnen werden, auch veröffentlicht werden, damit diese dann Eingang in die Fachcommunity finden können. Hierzu ist es nötig, dass man die gemachten Aussagen mit entsprechendem Bildmaterial unterlegt. Zudem wirkt ein Beitrag ohne diese - zum Teil außerordentlich kunstvollen - Karten wie eine Bleiwüste, denn wir erfreuen uns schließlich an den Illustrationen zum Text. Bedauerlich ist es, wenn die Autorinnen oder Autoren sich nicht nur mühevoll um die Digitalisierung dieser Kartenzimelien, sondern auch um nutzungsrechtliche Freigaben (Bildrechte) kümmern müssen, die selbst in staatlichen Archiven zumeist mit hohen Kosten bei einer etwaigen Veröffentlichung einhergehen.

Unser Autor in dieser Ausgabe, Thomas Horst, dem wir auch den Vorschlag zu dem Titelbild zu verdanken haben, musste diese leidvolle Erfahrung machen. Er hatte für seinen Beitrag zur Pfalz-Neuburgischen Landesaufnahme (ab S. 301) zunächst andere Abbildungen vorgesehen, für deren Digitalisierung bereits von einer von Heimatforschern initiierten Forschungsgruppe bezahlt wurde. Diese Bilder konnten dann aber aufgrund von Archivbereitstellungskosten, die für unsere Begriffe für eine nicht kommerzielle Nutzung zu hoch sind, nicht verwendet werden. Auch ein Schreiben der Schriftleitung der KN konnte das betreffende Archiv nicht umstimmen, auf die Kosten zu verzichten. Zum Glück gibt es jedoch auch einige private Institutionen (wie historische Vereine etc.), die ebenso bedeutendes historisches Kartenmaterial verwahren und ein besonderes Interesse daran haben, dieses der Öffentlichkeit zur Verfügung zu stellen.

Neben dem Artikel von Thomas Horst können Sie in dieser Ausgabe einen Beitrag von Werner Stams und Marianne Stams über das Lebenswerk von Max Eckert (1868-1938) lesen. Auch wenn wir uns in KN 3/17 bereits mit diesem bedeutenden Kartographen beschäftigt haben, können wir weitere Einblicke in das spannende Wirken von Max Eckert gewinnen. Der Beitrag von Thomas Büche und Kilian Loesch beschäftigt sich mit der automatisierten, GIS-gestützten Ableitung von Flussterrassen aus Geländemodellen und deren Darstellung im Kartenbild. Es würde mich freuen, wenn wir durch diesen und weitere Beiträge den Kreis der Autorinnen und Autoren und der Leserinnen und Lesern der KN auch innerhalb der geographischen Community erweitern können.

Ich wünsche Ihnen besinnliche Feiertage und einen guten Rutsch ins Jahr 2018. Ich würde mich freuen, wenn wir uns auf dem Neujahrsempfang in Berlin am 18. Januar 2018 treffen könnten.

Herzlichst,

Ihr

Morvatter 